\title{
Interference of surface plasmons and Smith-Purcell emission probed by angle-resolved cathodoluminescence spectroscopy
}

\author{
Naoki Yamamoto, ${ }^{1, *}$ F. Javier García de Abajo, ${ }^{2,3}$ and Viktor Myroshnychenko ${ }^{4,5}$ \\ ${ }^{1}$ Physics Department, Tokyo Institute of Technology, Meguro-ku, Tokyo 152-8551, Japan \\ ${ }^{2}$ ICFO-Institut de Ciencies Fotoniques, Mediterranean Technology Park, 08860 Castelldefels (Barcelona), Spain \\ ${ }^{3}$ ICREA-Institució Catalana de Recerca i Estudis Avançats, Passeig Lluís Companys, 23, 08010 Barcelona, Spain \\ ${ }^{4}$ Institute of Electrical Engineering, University of Paderborn, Warburger Straße 100, D-33098 Paderborn, Germany \\ ${ }^{5}$ Instituto de Química-Física - CSIC, Serrano 119, 28006 Madrid, Spain
}

(Received 19 June 2014; revised manuscript received 8 March 2015; published 30 March 2015)

\begin{abstract}
We investigate the interplay between geometrical lattice resonances and surface plasmons mediating the emission of Smith-Purcell visible light via angle-resolved cathodoluminescence spectroscopy. We observe strong modulations in the dispersion curves of Smith-Purcell radiation (SPR) when they intersect the surface plasmons of silver gratings using a 200-kV transmission electron microscope. The decay of the plasmons away from the grating is directly probed by controlling the electron-beam position relative to the sample surface with nanometer precision. Our measurements are in excellent agreement with numerical simulations, clearly revealing the presence of characteristic Fano profiles resulting from the interference of the light continuum and the discrete plasmon states for each direction of emission. The intensity anomaly in the SPR emission pattern can be well explained from the geometrical consideration of the intersections between the dispersion planes of the SPR and surface plasmon polariton (SPP). A strong and directional SPR beam can be realized under the condition that the SPR dispersion plane comes in contact with the band edge of the SPP dispersion plane.
\end{abstract}

DOI: 10.1103/PhysRevB.91.125144

PACS number(s): 78.60.Hk, 42.72.-g, 78.67.-n

\section{INTRODUCTION}

Smith-Purcell radiation (SPR) [1] is produced when an electron moves parallel to a grating as a result of the interaction between the evanescent electromagnetic field of the electron and the periodic corrugation of the grating. In a Huygens-like picture, light is emitted along directions of constructive coherent superposition, taking into account the delay in the signal produced from consecutive grooves due to the finite electron velocity. The wavelength of SPR continuously changes with emission angle, and thus, it holds great potential for tunable light sources in a wide spectral range from the UV to the $\mathrm{THz}$ domains. This effect can be explained from classical electrodynamics, describing the electron as a point charge [2-5]. An interesting extension is observed when the beam is bunched, so that several electrons are placed together within a small region compared with the emitted light wavelength [6-10]. The SPR intensity is then proportional to the squared number of electrons, in contrast to conventional uncorrelated SPR, whose intensity scales linearly with the electron current. This superradiant emission is an underlying principle of free-electron lasers and it can be exploited to produce intense $\mathrm{THz}$ emission [7], in good agreement with theoretical calculations [11,12]. SPR emission has been also observed from photonic $[13,14]$ and plasmonic $[15,16]$ crystals with periods of the order of the light wavelength.

In contrast to the extensively investigated $\mathrm{SPR}$ in the $\mathrm{THz}$ domain, only a limited effort has been devoted to studying SPR in the visible region [17-19]. In metallic gratings, one expects the emission intensity to be enhanced by the excitation

*yamamoto.n.ac@m.titech.ac.jp of surface plasmon polaritons (SPPs), which should provide increased coupling to the incident electrons. The spectral and electron-velocity dependence of the resulting emission has been recently analyzed [20]. In order to generate SPR with sufficient intensity, the electron beam needs to move close to the metal surface, within a short distance compared with the light wavelength. Under these conditions, one would expect a strong interference between the SPR emission and the excitation of SPPs, similar to what has been described in the literature for the radiative decay of SPPs in periodically modulated metal surfaces [21-24].

In this paper, we report the observation of strong interference between SPPs and SPR emission from one-dimensional (1D) silver gratings operating in the visible region. We present angle-resolved cathodoluminescence (CL) spectra acquired with a scanning transmission electron microscope (STEM) using electrons at various energies (from 90 to $200 \mathrm{keV}$ ) that are focused within a $\sim 10-\mathrm{nm}$ beam near the sample. Our measurements are performed in the aloof configuration, with the beam passing outside the grating at a controlled distance from its surface. Emission dispersion diagrams, collected as a function of emission direction and light energy, display characteristic SPR profiles and strong interference with the SPPs of the sample, including Fano profiles. Our results are supported by boundary-element method (BEM) [25] numerical simulations in good agreement with experiment.

The paper is organized as follows. We first describe some details of the experimental measurements used to study the silver grating system in Sec. II. Then, we present and discuss the results of our experimental and numerical studies of the emission spectra for the gratings of different periods taken at various accelerating voltages in Sec. III. Finally, the main conclusions are summarized in Sec. IV. 
(a)

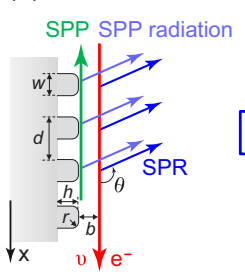

(b)

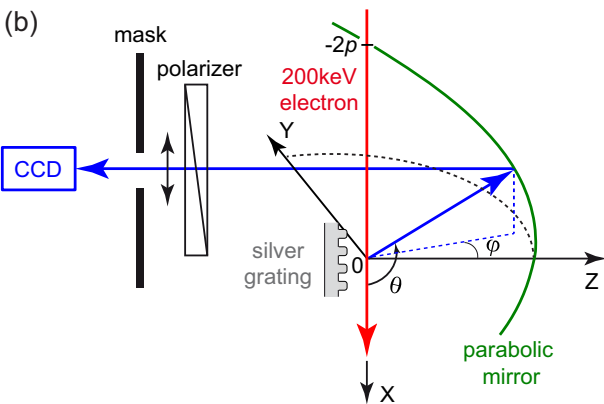

FIG. 1. (Color online) (a) Illustration of a 1D silver grating and an aloof electron moving parallel to its surface with impact parameter $b$. (b) Geometry of the angle-resolved measurement of Smith-Purcell radiation using a parabolic mirror. The specimen faces the mirror with its surface normal oriented parallel to the paraboloid axis $z$. The electron beam is incident along $x$. The cathodoluminescence (CL) emission is collected as a function of emission direction defined by angles $\theta$ and $\varphi$ through a moving pinhole mask.

\section{EXPERIMENTAL SETUP}

The specimens consist of $1 \mathrm{D}$ InP gratings of periods 500, 600 , and $800 \mathrm{~nm}$ patterned through electron-beam lithography and covered with a 200-nm silver layer evaporated in vacuum. A schematic cross section of the gratings is shown in Fig. 1(a). The groove width is half the period and the depth is $350 \mathrm{~nm}$ in all cases. The specimens are oriented in the STEM with the electron beam nearly parallel to their surface and perpendicular to the grooves. As the sample length is $\sim 1 \mathrm{~mm}$, it is slightly tilted $\left(<1^{\circ}\right)$ to allow the electron to interact with a sufficient number of grating periods close to one of the ends of the sample surface, while minimizing the fraction of beam electrons directly colliding with the metal. The microscope is equipped with a parabolic mirror placed above the specimen and adjusted to have its focus at the region of beam-grating interaction [see Fig. 1(b)]. The mirror used is $10 \mathrm{~mm}$ in width and $5 \mathrm{~mm}$ in height. A polarizer selects light emitted with its electric field perpendicular to the grooves. The emission measured with the orthogonal polarization (not shown) is featureless and comparatively much weaker. In what follows, we refer to the axes notation shown in Fig. 1(b) and define $\theta$ and $\varphi$ as the emission angles relative to $x$ (the beam direction) and $z$ (the grating normal) axes, respectively. For an angle-resolved measurement, the light emitted from the sample is collected by a charge-coupled device (CCD) detector, with the direction specified by angles $\theta$ and $\varphi$ selected through a movable mask, that is located between the polarizer and CCD detector and can be moved in a plane perpendicular to the $z$ axis $(X Y$ stage), and perforated by a small hole (solid angle $1.2 \times 10^{-2}$ sr at $\theta=180^{\circ}$ and $5 \times 10^{-2}$ sr at $\theta=90^{\circ}$ ). A single lens is used for transforming an emission image on the mirror into a mask plane. In order to measure the emission intensity pattern of the angle-resolved spectrum (ARS) which shows variation with emission angle $\theta$ and photon energy $E$, i.e., $I(E, \theta)$, the mask is moved vertically along the $x$ axis under the condition of $\varphi=0^{\circ}$. In this case, the differential intensity $d^{2} I / d \omega d \theta$ of the collected angle-resolved spectra is corrected for the
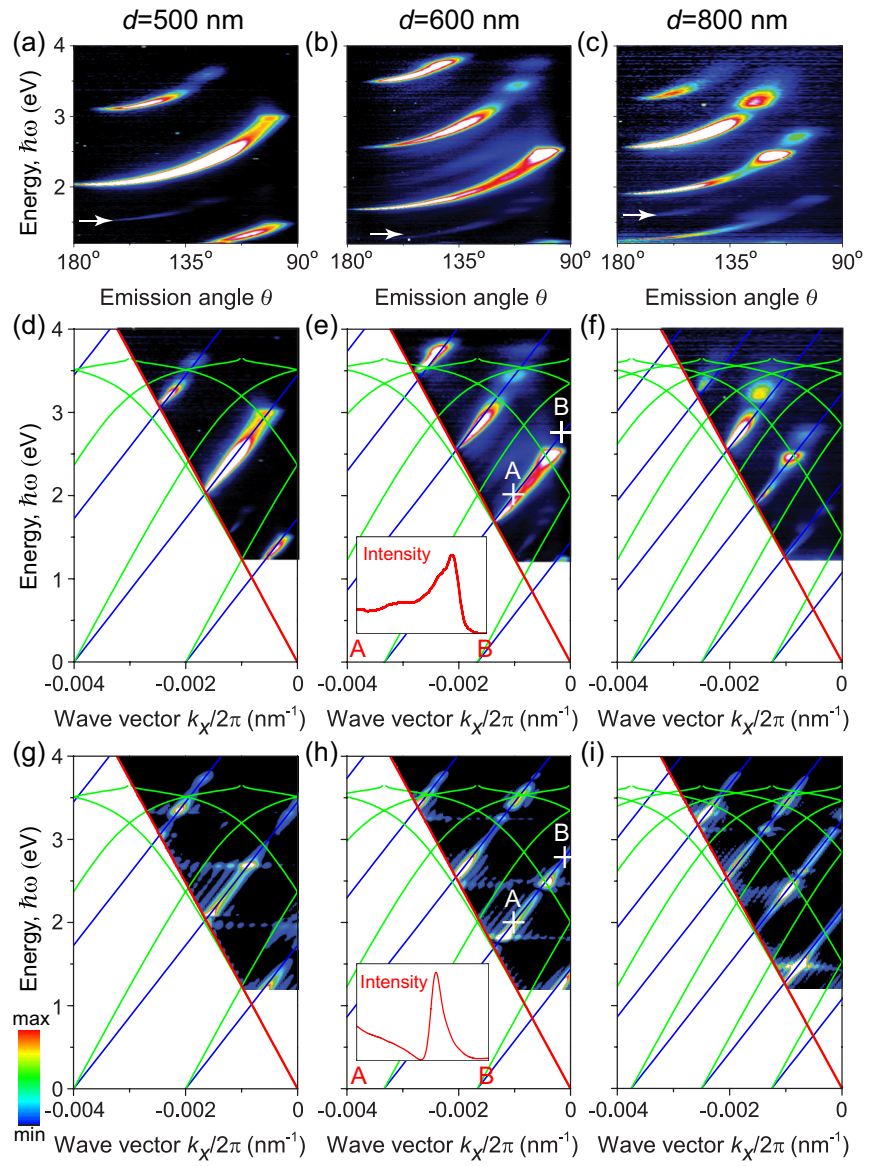

FIG. 2. (Color online) (a)-(c) Angle and energy dependence of the CL differential intensity $d^{2} I / d \omega d \theta\left(\varphi=0^{\circ}\right)$ originating from silver gratings of periods $d=500,600$, and $800 \mathrm{~nm}$. The $200-\mathrm{keV}$ electron is passing $\sim 50 \mathrm{~nm}$ away from the metal surface. (d)-(f) Dispersion patterns $d^{2} I / d \omega d k_{x}$ obtained by changing the angle $\theta$ in (a)-(c) to the wave vector $k_{x}=(\omega / c) \cos \theta$. (g)-(i) Calculated patterns corresponding to (d)-(f). Blue lines in (d)-(i) indicate the electron dispersion $k_{x}=v / c$ and its umklapp images displaced by an integral number of times $2 \pi / d$ along $k_{x}$. The green curves represent the surface plasmon dispersion of flat silver and its umklapp images. The red line indicates the dispersion line of light (the light line). Insets to (e) and (h) show experimental and theoretical CL intensity profiles along the $A B$ segments of the density plots.

dependence of detection efficiency on photon energy $\hbar \omega$. The spectral resolution of the CCD detector is typically $2 \mathrm{~nm}$ in wavelength.

\section{RESULTS AND DISCUSSION}

Figures 2(a)-2(c) shows ARS patterns acquired from gratings with different spacings $d$, clearly exhibiting pronounced intensity maxima piling up near the regions determined by SPR coherence (see below). The data are then replotted as dispersion diagrams $\left[d^{2} I / d \omega d k_{x}\right.$, Figs. 2(d)-2(f)] by transforming the emission angle $\theta\left(\varphi=0^{\circ}\right)$ into the surfaceparallel wave-vector component $k_{x}=(\omega / c) \cos \theta$, and also multiplying the intensity by the Jacobian of this transformation. The observed emission maxima, collected for $k_{x}<0$, are 
in excellent agreement with the condition of SPR coherence [Figs. 2(d)-2(f), blue lines]

$$
\omega=v\left(k_{x}+\frac{2 \pi n}{d}\right),
$$

where $\omega$ is the light frequency, $d$ is the grating period, $v$ $(=0.7 c$ at $200 \mathrm{keV})$ is the electron velocity, and the emission order $n$ indicates the number of optical periods that separate the signal produced from consecutive grooves. Different orders are clearly observed in each dispersion plot.

Surface plasmon polaritons can be excited by the incident electron beam when it passes near the metal surface in the aloof configuration. The excitation of SPPs may affect the SPR emission. In order to explore this phenomenon, we consider the SPP dispersion relation in a flat silver surface [26] $\pm k_{x}=$ $(\omega / c) \sqrt{\epsilon /(\epsilon+1)}$, where $\epsilon$ is the metal dielectric function. Scattering of SPPs by the grating can add momentum to the left-hand side of this equation in units of $2 \pi / d$. In the limit of weak scattering, this leads to an infinite series of SPP dispersion curves separated along $k_{x}$ by multiples of $2 \pi / d$ and shown in Figs. 2(d)-2(f) as green curves. The actual SPP dispersion curves will be corrected by dynamical interaction effects such as gap openings at the Brillouin-zone boundaries, but the weak-scattering limit provides an intuitive tool to understand the experiment. Indeed, as we move along each SPR emission line (blue), we observe strong intensity modulations near the crossings with SPP curves (green), thus revealing the presence of strong interaction between SPPs and SPR.

In order to understand the SPP-SPR interaction, we perform BEM simulations corresponding to the geometry depicted in Fig. 1 with parameters mimicking those of the experimental geometry (impact parameter $b=10 \mathrm{~nm}$, groove depth $h=$ $350 \mathrm{~nm}$, and ridge edge rounding radius $r=25 \mathrm{~nm}$ ), and the dielectric function of silver taken from optical data [27]. We consider a grating consisting of ten periods. The calculated intensity distributions [Figs. 2(g)-2(i)] are in reasonable agreement with experiment [Figs. 2(d)-2(f)], including the presence of strong modulations along SPR lines. Incidentally, the computed intensity decreases by a factor of 3 when looking at the light emitted with polarization along the grooves (not shown).

The modulations produced by SPPs in the SPR emission can be understood in terms of Fano interference [13,28]. A system formed by a discrete state that is coupled to a continuum of states can be diagonalized exactly to produce a modified continuum, whose excitation follows a family of universal Fano profiles that are characterized by a dip-peak structure near the energy of the discrete state [28]. In our case, when considering the emission along one of of SPR lines of the dispersion diagrams, the continuum consists of the propagating photon states along that line, whereas the discrete state is supplied by the SPP mode that crosses the SPR line along a single point. We present an example of Fano interference in Figs. 2(e) and 2(h), where we represent the intensity along the $A B$ line of the density plots (insets). A dip-peak asymmetric Fano profile is clear in the theory curve and also visible in the experimental one, which is however affected by surface imperfections and the beam profile near the sample.
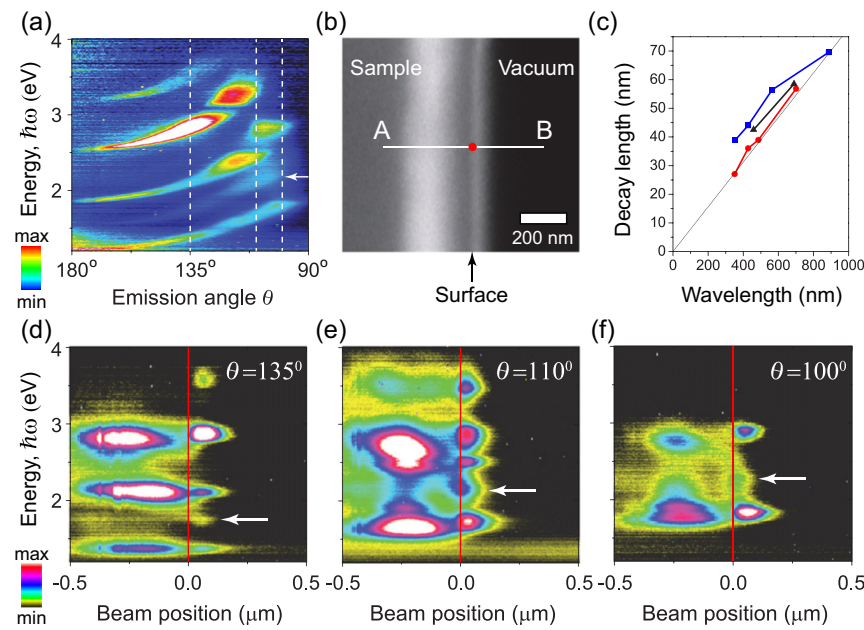

FIG. 3. (Color online) (a) Angle- and energy-resolved CL emission from the same $d=800 \mathrm{~nm}$ grating as in Fig. 2(c), taken with an electron beam located close to the surface. (b) Panchromatic map of the sample, revealing the metal surface and the depth of the grating $(\sim 350 \mathrm{~nm})$. (c) Wavelength dependence of the CL decay length with electron-surface separation. (d)-(f) Spectral images taken at fixed emission angles (d) $\theta=135^{\circ}$, (e) $\theta=110^{\circ}$, and (f) $\theta=100^{\circ}$, marked by vertical white lines in (a). The horizontal axis indicates the electron-beam position relative to the metal surface (vertical line). The data in (c) are obtained by analyzing (d) (squares), (e) (circles), and (f) (triangles).

Figure 3(a) shows an ARS pattern from the $d=800-\mathrm{nm}$ grating taken with an incident beam located close to the metal surface. This reveals the same features as in the aloof pattern of Fig. 2(c) plus an additional faint contrast (white arrow) that is parallel to the SPR dispersion lines and is located at the central position between the neighboring SPR lines, presumably the SPR produced by the interference of radiations from the double-period modulation of the grating. Similar contrasts are also seen in Figs. 2(a)-2(c) as indicated by white arrows. A panchromatic, omnidirectional CL image [Fig. 3(b)], constructed by rastering the beam over the sampled area, shows that the photon emission quickly drops when moving towards the vacuum side, to the right of the incident beam position used in Fig. 3(a), which is marked by a dot. We explore the spectral distribution of this drop in emission by collecting ARS as a function of beam position along the $A B$ segment of Fig. 3(b). The results are shown in Figs. 3(d)-3(f) for three different directions of emission, indicated by vertical dashed lines in Fig. 3(a). The additional faint contrast appears as indicated by a white arrow in Fig. 3(a). The additional feature due to the double-period SPR noted above is indicated by white arrows at energy positions that are consistent with Fig. 3(a). Their intensity distribution is localized near the surface. In all cases, the emission peaks are rather independent of beam position. Interestingly, electrons traversing the groove edges (negative beam positions) still produce SPR emission, with a depletion observed right at the surface, possibly connected to the slight tilting of the sample mentioned above.

In aloof interaction (beam above surface), the intensity decays exponentially with beam-surface distance $z$. The decay 
(a) $1.92 \mathrm{eV}$

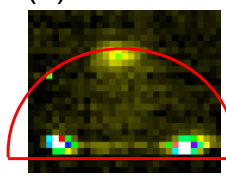

(e) $2.2 \mathrm{eV}$

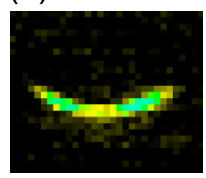

(i) $2.7 \mathrm{eV}$

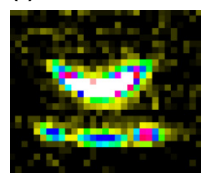

(b) $1.95 \mathrm{eV}$

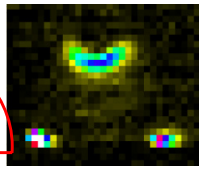

(f) $2.3 \mathrm{eV}$

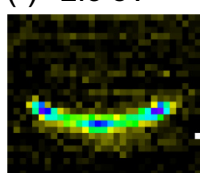

(j) $2.8 \mathrm{eV}$

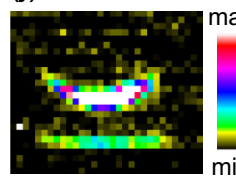

(c) $2.0 \mathrm{eV}$

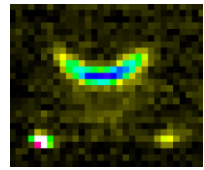

(g) $2.4 \mathrm{eV}$

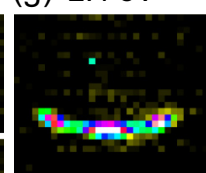

(d) $2.1 \mathrm{eV}$

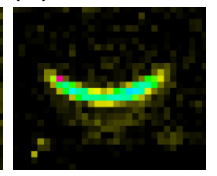

(h) $2.6 \mathrm{eV}$

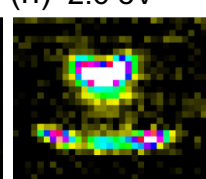

(k)

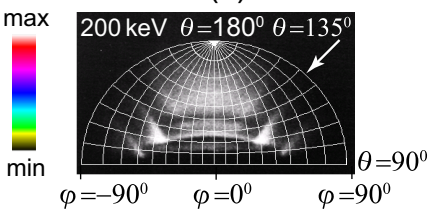

FIG. 4. (Color online) (a)-(j) Monochromatic emission patterns from the $d=800$-nm grating taken at $200 \mathrm{keV}$ by scanning the pinhole mask in the mirror image plane, presented at various photon energies from (a) $1.92 \mathrm{eV}$ to (j) $2.8 \mathrm{eV}$. (k) The panchromatic emission pattern observed by the CCD camera under the same condition, together with the contour lines of the emission angles $(\theta, \varphi)$.

length $L$ is represented in Fig. 3(c), as extracted from the energy features observed in Fig. 3(d) (squares), Fig. 3(e) (circles), and Fig. 3(f) (triangles) [i.e., the intensity is fitted by a function $e^{-\left(z-z_{0}\right) / L}$ to the right of the maximum at $z=z_{0}$ ]. This effect is due to the exponential drop in the electromagnetic field intensity produced by the electron, which extends a characteristic distance [29]

$$
L=\frac{\gamma v}{2 \omega}=\frac{\lambda}{4 \pi} \frac{1}{\sqrt{c^{2} / v^{2}-1}}
$$

from the beam, where $\gamma$ is the Lorenz contraction factor, which cannot be neglected at the $200-\mathrm{keV}$ energies used in the experiment $(\gamma=1.4)$. This linear relation between the light wavelength $\lambda$ and $L$ is in excellent agreement with experiment, as show in Fig. 3(c) (solid line).

Figures 4(a)-4(j) shows monochromatic emission patterns from the $d=800-\mathrm{nm}$ grating taken at $200 \mathrm{keV}$ by scanning the pinhole mask in the mirror image plane, presented at various photon energies from (a) $1.92 \mathrm{eV}$ to (j) $2.8 \mathrm{eV}$. The emission pattern corresponds to the SPR of the order of $n=-3$. In Figs. 4(h)-4(j), the emission pattern due to the SPR of the order of $n=-4$ also appears at the higher angle position. Figure $4(\mathrm{k})$ shows the panchromatic emission pattern observed by the CCD camera under the same condition, together with the contour lines of the emission angles $(\theta, \varphi)$. In the monochromatic emission patterns, the emission intensity appears along the contour line of the polar angle $\theta$ (downward arc-shaped contrasts). The emission starts to appear at $\theta=180^{\circ}$ in Fig. 4(a), shifting to the lower angles with increasing energy, and gradually disappears after reaching the contour at $\theta=90^{\circ}$. On the other hand, in Fig. 4(k), upward arc-shaped contrasts and streak contrasts appear in the range $\theta$ from $135^{\circ}$ to $90^{\circ}$.

The interference condition for the SPR can be written as

$$
\left(\boldsymbol{k}_{e}-\boldsymbol{k}\right) \cdot \boldsymbol{d}=2 n \pi,
$$

(a)

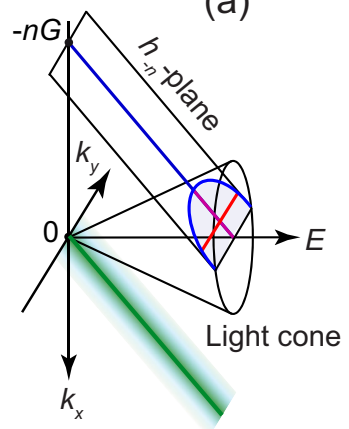

(d)

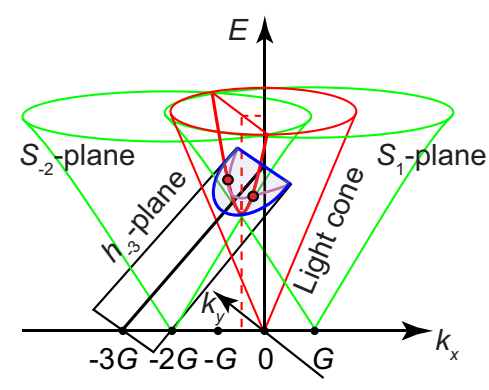

(b)

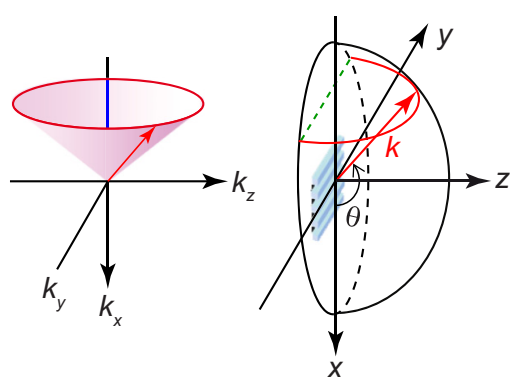

(e)

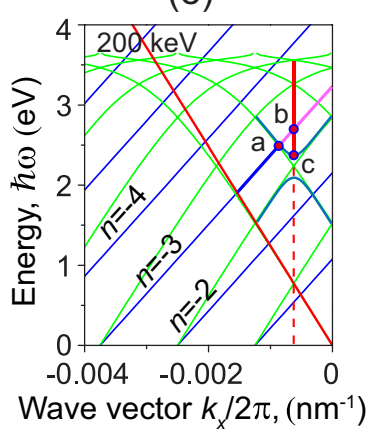

FIG. 5. (Color online) (a) Schematic representation of the dispersion plane $\left(h_{n}\right.$ plane) of the $n$th SPR in the $E-\boldsymbol{k}$ space. The observable backward emission occurs on the dispersion plane inside the light cone indicated by a blue color. (b) The cone plane in the $\boldsymbol{k}$ space formed by the SPR wave vectors with equal photon energy. (c) Emission directions of the SPR with equal photon energy in a real space. (d) The configuration of the SPR dispersion plane $\left(h_{-3}\right.$ plane) and the SPP dispersion planes in the $E-\boldsymbol{k}$ space. The intensity anomaly of the SPR arises along the intersection line (pink) and especially at the spots (red) on the band edge line (red) at the zone boundary. (e) Dispersion lines of the SPR and SPP on the $E-k_{x}$ cross-sectional plane in (d) for the $d=800$-nm grating and $200-\mathrm{keV}$ incident beam.

where $\boldsymbol{k}$ is the wave vector of the emitted light, $\boldsymbol{k}_{\boldsymbol{e}}$ is the wave vector parallel to the electron trajectory with a magnitude of $\omega / v$, and $\boldsymbol{d}$ is the vector parallel to the $x$ axis with a magnitude of the period $d$. Figure 5(a) illustrates the dispersion plane $\left(h_{n}\right.$ plane) of the $n$th SPR in the $E-\boldsymbol{k}$ space, where the emission occurs on the dispersion plane inside the light cone indicated by blue color. The SPRs with an equal energy arise along the red line in the blue region. If the photon energy of the SPR is the same, i.e., $\omega=$ const, it follows that

$$
k_{x}=k_{e}-\frac{2 n \pi}{d}=\frac{\omega}{v}-n G
$$

and thus the $x$ component of $\boldsymbol{k}, k_{x}$, is constant. The wave vectors with constant $k_{x}$ are placed on the cone plane in the $\boldsymbol{k}$ space as shown in Fig. 5(b). This means that the SPR with constant energy emits to the directions of the constant polar angle $\theta$ in the real space as shown in Fig. 5(c), which produces the downward arc-shaped contrast in the monochromatic emission pattern in Fig. 4. The intensity changes monotonically along the arc in the range of $\theta$ from 
$180^{\circ}$ to $135^{\circ}$, where the SPR shows rather homogeneous distribution in the panchromatic emission pattern in Fig. 4(k).

In the range of $\theta$ from $135^{\circ}$ to $90^{\circ}$, the intensity distribution along the arc becomes inhomogeneous as seen in Figs. 4(e)4(j). The intensity modulation along the arc arises at the cross positions with the downward arc-shaped contrasts and the streak contrasts seen in Fig. 4(k). This modulation is considered to be attributed to the interaction between the SPR and SPP as mentioned before. Figure 5(d) represents the configuration of the SPR dispersion plane $\left(h_{-3}\right.$ plane) and the SPP dispersion plane in the $E-\boldsymbol{k}$ space. The dispersion plane of the SPP in the periodic structure can be approximated by a set of dispersion planes obtained from a single coneshaped dispersion plane of the SPP on a flat surface by shifting the SPP dispersion cone by the reciprocal-lattice vectors (the empty lattice approximation) [24]. Two dispersion planes emerging from the $G$ and $-2 G$ reciprocal-lattice points $\left(S_{1}\right.$ and $S_{-2}$ planes) are drawn in Fig. 5(d) together with the light cone colored in red. The curve generated by the intersection of the $h_{-3}$ dispersion plane of the SPR and the SPP dispersion plane emerging from the $G$ point (the $S_{1}$ plane) is indicated by a pink line, which corresponds to the upward arc-shaped contrast in Fig. 4(k). Figure 5(e) shows the dispersion curves of the SPR and SPP on the $E-k_{x}$ cross-sectional plane in Fig. 5(d) for the $d=800-\mathrm{nm}$ grating and 200-keV incident beam. The calculated value of the polar angle is $\theta=118.6^{\circ}$ at $\varphi=0^{\circ}$ on the intersection line [the position $a$ in Fig. 5(e)], that is in good agreement with the position of the upward arc-shaped contrast in Fig. 4(k). The other upward arcs in Fig. 4(k) can correspond to the intersection of the $S_{1}$ plane with the SPR dispersion planes of different order.

The $S_{1}$ and $S_{-2}$ planes produce a band gap along their intersection line (the red curve) at the Brillouin-zone boundary $\left(k_{x}=-G / 2\right)$ as shown in Fig. 5(d). Strong interaction with the SPR can be expected to occur at the band edge because of the high density of states (DOS) of the SPP. Such positions are indicated by red spots at the crossing points of the pink and red curves in Fig. 5(d), corresponding to the position $b$ in Fig. 5(e). The calculated values of these positions are $(\theta, \varphi)=\left(106.4^{\circ}, \pm 45.7^{\circ}\right)$, which coincide with the positions of the strong streak contrast in Fig. 4(k). The streak pattern is considered to arise along the intersection between the SPR dispersion plane and the SPP dispersion plane (the $S_{-2}$ plane) near the band edge. These facts clearly indicate that the intensity modulation along the SPR dispersion line originates from the interaction between the SPR and SPP.

The inclination of the SPR dispersion plane in the $E-\boldsymbol{k}$ space changes with velocity of the incident electron according to Eq. (1). The inclination angle decreases with decreasing an accelerating voltage, which causes the intersecting position of the SPR and SPP dispersion planes and changes emission pattern. Figures 6(a)-6(f) shows panchromatic emission patterns (the upper row) from the $d=800$-nm grating taken at various accelerating voltages by using a CCD camera to image the parabolic mirror. The lower row in Figs. 6(a)-6(f) shows corresponding ARS patterns at $\varphi=0^{\circ}$ acquired in the same way as before. The upward arc-shaped contrasts and the streaks are seen to change positions with varying an accelerating voltage. The upward arcs shift to the lower polar angles and the streaks move to the central position $\left(\varphi=0^{\circ}\right)$

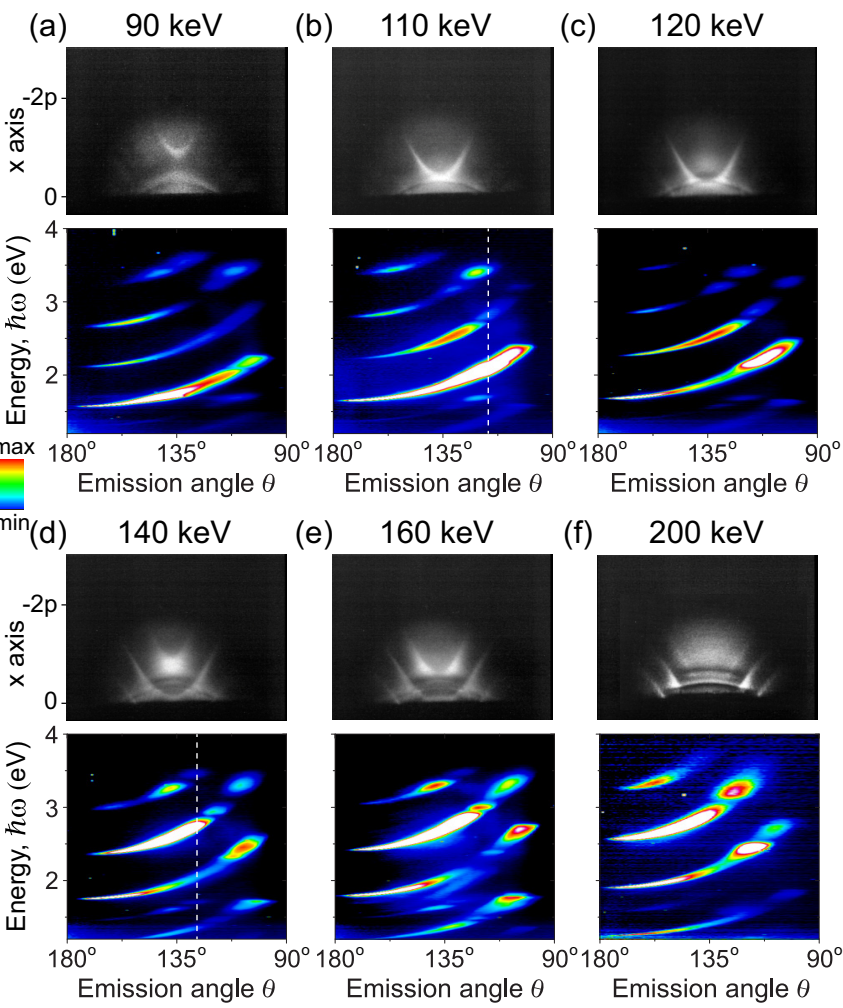

FIG. 6. (Color online) (a)-(f) Panchromatic emission patterns (the upper row) from grating of period $d=800 \mathrm{~nm}$ obtained by imaging the parabolic mirror using the CCD camera taken at various accelerating voltages. The lower row shows corresponding ARS patterns at $\varphi=0^{\circ}$ acquired in the same way as in Fig. 2. The strong directional beam appears in (b) $110 \mathrm{keV}$ and (d) $140 \mathrm{keV}$.

as the accelerating voltage decreases. Correspondingly, the intensity modulation along the SPR dispersion curves is seen to occur. It is noted that when the streaks intersect at the central position, they produce a strong and directional beam at $110 \mathrm{keV}$ [Fig. 6(b)] and $140 \mathrm{keV}$ [Fig. 6(d)].

Figure 7 shows dispersion patterns from the $d=800-\mathrm{nm}$ grating at (a) $110 \mathrm{keV}$ and (b) $140 \mathrm{keV}$ in $E-k_{x}$ plane transformed from the ARS patterns in Figs. 6(b) and 6(d). In Fig. 7(a) the SPR dispersion plane of $n=-3$ (the $h_{-3}$ plane) intersects the crossing point of the $S_{1}$ and $S_{-2}$ SPP dispersion planes (red arrow); the position corresponds to the band edge as indicated by the point $c$ in Fig. 5(e). The polar angle at the point $c$ is calculated to be $\theta=110.4^{\circ}$, which is in good agreement with the strong beam position in Fig. 6(b). In Fig. 7(b), the SPR dispersion plane of $n=-4$ (the $h_{-4}$ plane) intersects the crossing point of the $S_{1}$ and $S_{-3}$ SPP dispersion planes, the position of which corresponds to the polar angle of $\theta=123.9^{\circ}$ and coincides with the position of the directional beam in Fig. 6(d). These facts indicate that the SPR is enhanced as a strong and directional beam under the condition that the SPR dispersion plane comes in contact with the SPP band edge at the Brillouin-zone boundary.

The observed intensity modulation along the SPR dispersion line originates from the interaction between the SPR and the backward SPPs such as those along the $S_{1}$ dispersion line in Fig. 2. The previous theories of the SPR $[4,30]$ predicted 
(a)

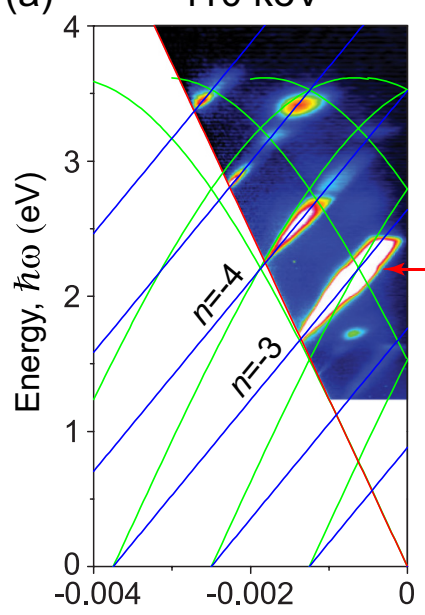

Wave vector $k_{X} / 2 \pi\left(\mathrm{nm}^{-1}\right)$

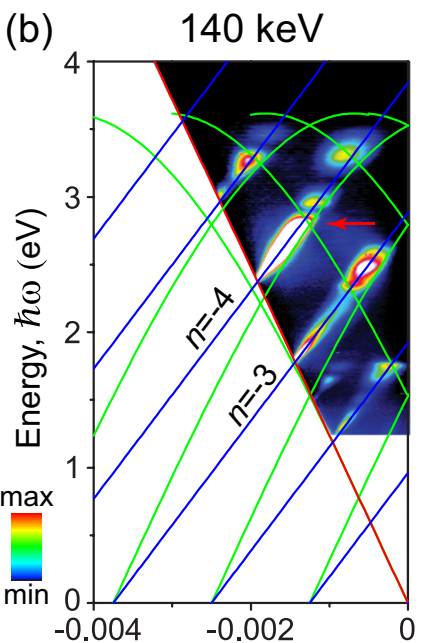

Wave vector $k_{x} / 2 \pi\left(\mathrm{nm}^{-1}\right)$

FIG. 7. (Color online) Dispersion patterns from the $d=800-\mathrm{nm}$ grating at (a) $110 \mathrm{keV}$ and (b) $140 \mathrm{keV}$ in $E-k_{x}$ plane transformed from the ARS patterns in Figs. 6(b) and 6(d). Intensity enhancement occurs near the crossing point of the two SPP dispersion lines, when the SPR dispersion plane comes in contact with the band edge at the crossing point.

only the anomaly in the forward directions of $0^{\circ}<\theta<90^{\circ}$ due to the interaction with the forward SPPs, because the incident electron can directly excite SPPs propagating in the forward direction. However, such emission anomaly was not observed in the backward emission patterns in Figs. 2 and 7; the emission induced by the excited SPP through the metal grating should occur along the SPP dispersion lines (the green lines in Figs. 2 and 7), though such emission is not seen in the figures. One reason is that the emission from the SPP in the 1D plasmonic crystal with a rectangle cross section becomes weak when the corrugation height exceeds $200 \mathrm{~nm}$ [31]. In addition, there is very small probability for the aloof incident electron to directly excite the backward SPP in the grating, so the emission along the $S_{1}$ dispersion

line should not appear as was observed in Figs. 2 and 7. Only the emission along the dispersion lines of the forward SPPs shifted by the reciprocal-lattice vectors such as the $S_{-2}$ dispersion line can contribute to the backward emission. In fact we observed the SPP induced emission along the $S_{-2}$ dispersion line using a grating with a corrugation height of $100 \mathrm{~nm}$, though the emission along the $S_{1}$ dispersion line was not observed. Therefore we can conclude that the excitation of the SPR by the incident electron is accompanied by the interaction with the backward SPP which is simultaneously excited at the crossing point of their dispersion lines. The BEM calculation treats this process correctly as it well reproduces the intensity anomaly along the SPR dispersion lines.

\section{CONCLUSIONS}

In conclusion, we have observed that the interaction between SPPs and SPR leads to Fano profiles along the direction of constructive emission, characterized by a dip-peak structure, in good agreement with electromagnetic simulations. In the aloof configuration, with the electron moving at a distance from the grating surface, the electron is still capable of interacting with the grating, and the predicted decay length of the electromagnetic intensity of the electron $\sim \gamma v / 2 \omega$ is shown to be in excellent agreement with experiment. The intensity anomaly in the SPR is well represented in the monochromatic and panchromatic emission patterns, which can be explained from the geometrical consideration of the intersections between the dispersion planes of the SPR and SPP. A strong and directional SPR beam can be realized under the condition that the SPR dispersion plane comes in contact with the band edge of the SPP dispersion plane.

\section{ACKNOWLEDGMENTS}

The authors acknowledge support from the Japanese MEXT Nanotechnology Platform No. 120250214 and the Spanish MICINN (Grant No. MAT2010-14885). V.M. acknowledges support from CSIC through the JAE program.
[1] S. J. Smith and E. M. Purcell, Phys. Rev. 92, 1069 (1953).

[2] P. M. van den Berg, J. Opt. Soc. Am. 63, 689 (1973).

[3] P. M. van den Berg, J. Opt. Soc. Am. 63, 1588 (1973).

[4] O. Haeberlé, P. Rullhusen, J. M. Salomé, and N. Maene, Phys. Rev. E 49, 3340 (1994).

[5] Z. J. Shi, X. P. Tang, F. Lan, and Z. Q. Yang, J. Infrared Millimeter Waves 33, 183 (2014).

[6] L. Schachter and A. Ron, Phys. Rev. A 40, 876 (1989).

[7] J. Urata, M. Goldstein, M. F. Kimmitt, A. Naumov, C. Platt, and J. E. Walsh, Phys. Rev. Lett. 80, 516 (1998).

[8] D. Li, Z. Yang, K. Imasaki, and G.-S. Park, Phys. Rev. ST Accel. Beams 9, 040701 (2006).

[9] J. T. Donohue and J. Gardelle, Phys. Rev. ST Accel. Beams 8, 060702 (2005).

[10] Y. Shibata, S. Hasebe, K. Ishi, S. Ono, M. Ikezawa, T. Nakazato, M. Oyamada, S. Urasawa, T. Takahashi, T. Matsuyama et al., Phys. Rev. E 57, 1061 (1998).
[11] H. L. Andrews, C. H. Boulware, C. A. Brau, and J. D. Jarvis, Phys. Rev. ST Accel. Beams 8, 110702 (2005).

[12] H. L. Andrews, C. H. Boulware, C. A. Brau, and J. D. Jarvis, Phys. Rev. ST Accel. Beams 8, 050703 (2005).

[13] T. Ochiai and K. Ohtaka, Opt. Express 13, 7683 (2005).

[14] K. Ohtaka and S. Yamaguti, Opt. Spectrosc. (USSR) 91, 506 (2001).

[15] T. Ochiai and K. Ohtaka, Phys. Rev. B 69, 125107 (2004).

[16] F. J. García de Abajo and L. A. Blanco, Phys. Rev. B 67, 125108 (2003).

[17] A. S. Kesar, M. Hess, S. E. Korbly, and R. J. Temkin, Phys. Rev. E 71, 016501 (2005).

[18] S. Taga, K. Inafune, and E. Sano, Opt. Express 15, 16222 (2007).

[19] G. Adamo, K. F. MacDonald, Y. H. Fu, C. M. Wang, D. P. Tsai, F. J. García de Abajo, and N. I. Zheludev, Phys. Rev. Lett. 103, 113901 (2009). 
[20] J.-K. So, K. F. MacDonald, and N. I. Zheludev, Appl. Phys. Lett. 104, 201101 (2014).

[21] D. Heitmann, J. Phys. C 10, 397 (1977).

[22] J. T. van Wijngaarden, E. Verhagen, A. Polman, C. E. Ross, H. J. Lezec, and H. A. Atwater, Appl. Phys. Lett. 88, 221111 (2006).

[23] N. Yamamoto and T. Suzuki, Appl. Phys. Lett. 93, 093114 (2008).

[24] T. Suzuki and N. Yamamoto, Opt. Express 17, 23664 (2009).

[25] F. J. García de Abajo and A. Howie, Phys. Rev. B 65, 115418 (2002).
[26] H. Raether, Surface Plasmons on Smooth and Rough Surfaces and on Gratings, Springer Tracks in Modern Physics Vol. 111 (Springer-Verlag, Berlin, 1988).

[27] P. B. Johnson and R. W. Christy, Phys. Rev. B 6, 4370 (1972).

[28] U. Fano, Phys. Rev. 124, 1866 (1961).

[29] F. J. García de Abajo, Rev. Mod. Phys. 82, 209 (2010).

[30] S. L. Chuang and J. A. Kong, J. Opt. Soc. Am. A 1, 672 (1984).

[31] H. Watanabe, M. Honda, and N. Yamamoto, Opt. Express 22, 5155 (2014). 